\title{
Interruption of aortic arch without associated cardiac abnormalities
}

\author{
V. M. Judez, M. J. Maitre, M. de Artaza, J. M. F. de Miguel, F. Valles, and J. Marquez \\ From Clinica Puerta de Hierro, National Center for Medico-Surgical Research, \\ National Health Service, Madrid, Spain
}

The clinical and angiographic findings of a patient showing the features of a congenitally interrupted aortic arch, with no evidence of a persistent ductus arteriosus or any other intracardiac pathology, are described. The other three reported cases of this rare malformation, and the current hypothesis on its embryogenesis, are briefly reviewed.

Interruption of the aortic arch is an infrequent congenital anomaly. In its usual variety it is almost constantly associated with a persistent ductus arteriosus which provides adequate blood flow to the descending aorta. Coincidental cardiac malformations are often present, such as a ventricular septal defect or a bicuspid aortic valve, among many others.

Isolated absence or interruption of the aortic arch, without a ductus or any other cardiac defect is an exceptional finding. In our search through the published reports, we were able to trace only three previous reports of this pathology (Pillsbury, Lower, and Shumway, 1964; Zetterquist, 1967; Morgan et $a l .$, 1970), all of them diagnosed in life. We describe here the clinical and angiographic data of a patient fulfilling the criteria for the diagnosis of this rare condition.

\section{Case history}

An I8-year-old girl was referred to one of us ( $M$ de $A$ ) for consultation, with impalpable peripheral arterial pulses. She was born after an uneventful pregnancy, and no comments were made on her cardiac condition at the time of birth. Thereafter the patient was able to lead a normal and symptomless life. Attention to her absent peripheral pulses was paid in a routine physical examination in her adolescence.

She was a normally built young girl, with no cyanosis. The jugular venous pressure and pulse were normal. Both carotid arteries were easily felt, the pulse being full and bounding. No radial, brachial, or axillary pulses were felt in either arm. Femoral pulses were weak and late; popliteal and dorsalis pedis were absent bilaterally. Examination of the neck revealed the presence of bi- lateral nodular masses, about $3 \mathrm{~cm}$ in length, superficial, pulsating, and located anteriorly and medially to the sternocleidomastoid muscle.

The cardiac impulse was normal in character. A short ejection systolic murmur was heard at the left sternal border; the second sound split normally in inspiration. Otherwise the physical examination did not provide any further abnormal findings.

The electrocardiogram showed non-specific $T$ wave abnormalities in the left ventricular leads. No significant abnormalities were found in the routine laboratory tests. Examination of the optic fundi showed tortuous arteries and veins, but no hypertensive changes.

Chest $x$-ray films showed a normal cardiac outline, though no aortic knob was clearly identified. The pulmonary vascular pattern was normal. There was a deformity of the first left rib, but no costal erosions. A bilateral nodular shadow, of low density but neatly defined, was present at both lung apices. Its significance remained imprecise at that time (Fig. IA).

Digital plethysmography was consistent with a nonpulsatile pattern of blood flow in the four extremities.

A clinical diagnosis of coarctation of the aorta, with abnormal, postcoarctation, origin of the left subclavian artery, and an aberrant right subclavian artery was made.

Cardiac catheterization was performed (Table); approach to the left side of the heart was achieved through a left atrial transseptal puncture. No shunts were detected at any level. The intracardiac pressures were within normal limits. Though no arterial sample was obtained, the normal right heart pressures did not support a rightto-left shunt at pulmonary artery level.

Cineangiography (Fig. IB, C, D) showed a normal left atrium and ventricle; the aorta emerged in a straight upward direction, bifurcating into both common carotid arteries which were the only vessels arising from the ascending aorta. The lateral projection showed the complete absence of an aortic arch, though a slight posterior bending of the ascending aorta was apparent. 
TABLE Cardiac catheterization data

\begin{tabular}{lll}
\hline & $\begin{array}{l}\text { Pressures } \\
(\mathrm{mmHg})\end{array}$ & $\begin{array}{l}\text { Oxygen satn } \\
(\%)\end{array}$ \\
\hline Rt atrium & $\begin{array}{l}\text { a: } 7 \text { v: } 6 \\
\text { x: } 1 \text { y: } 3 \\
\text { mean: } 4\end{array}$ & 83 \\
Rt ventricle & $37 / 5$ & \\
Lt atrium & $\begin{array}{l}\text { a: } 10 \text { v: } 12 \\
\text { x: } 5 \text { y: } 7 \\
\text { mean: } 10\end{array}$ & 95 \\
Lt ventricle & 120/10 & 95 \\
\hline
\end{tabular}

No contrast reached the descending aorta at that time. Later, retrograde circulation from the head was detected, flowing through two different vascular systems: one consisted of the normal vertebral arteries; the other, of large and tortuous cervical vessels, which were better developed in the left side of the neck. The confluence of the vertebral and cervical systems on each side gave origin to a large vessel with a straight caudal and medial course; the fusion of these two dorsal aortae resulted finally in a descending aorta of normal size. Both subclavian arteries seemed to arise from the point of junction of the vertebral and cervical systems; they were thin and carried a small flow. At no time did the film indicate a persistent ductus. Only then was it possible to identify the pulmonary apical shadows, by the abnormal cervical vessels.
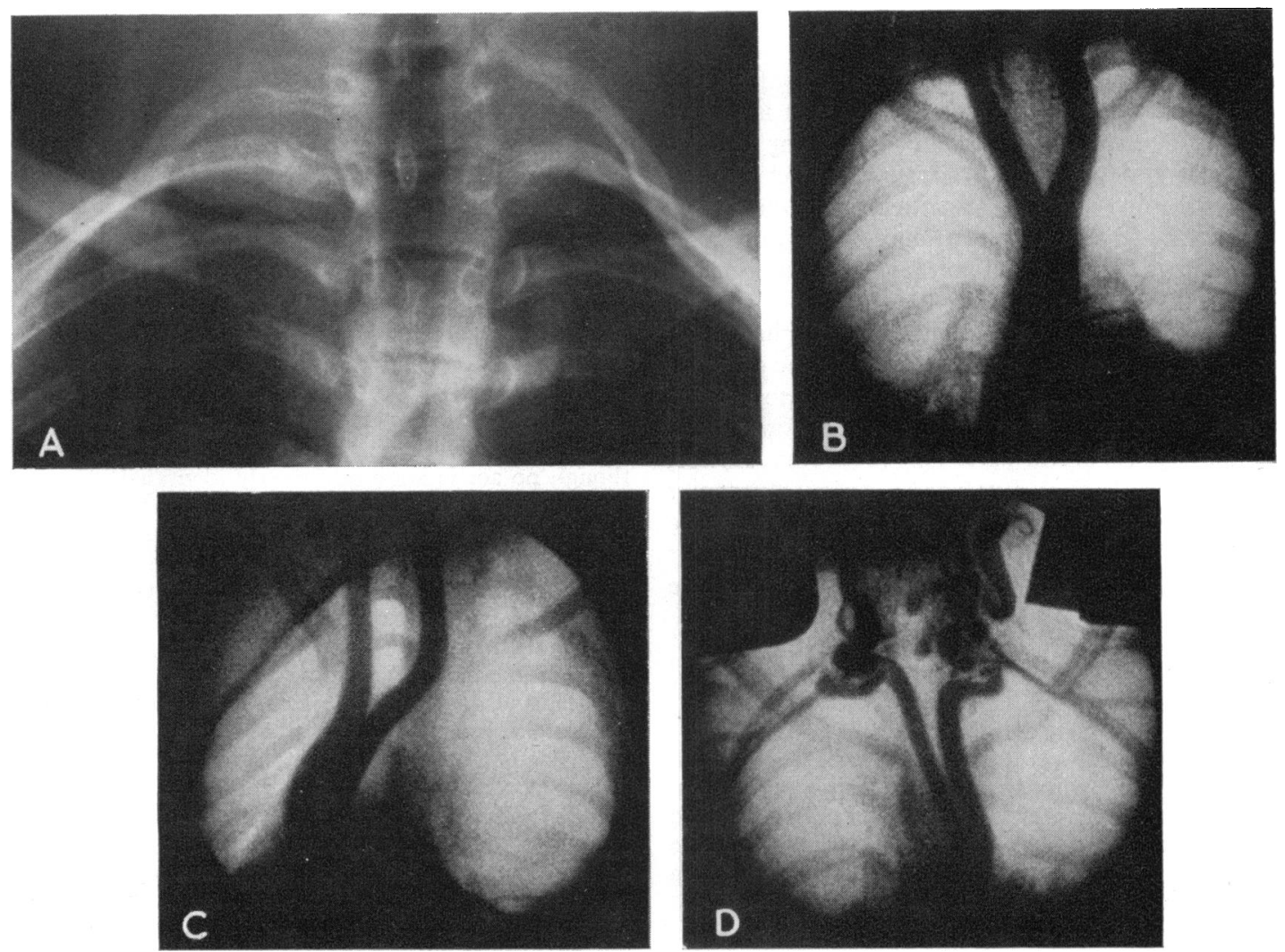

FIG. I (A) Chest $\mathrm{x}$-ray film showing abnormal shadows at both lung apices. (B) Cineangiogram, early frames: contrast medium opacifies both primitive carotid arteries which are the only branches from the aortic arch. $(C)$ Left oblique projection, showing slight posterior bending of the ascending aorta. $(D)$ Late frames: considerable retrograde flow through vertebral and cervical vessels opacifies two dorsal aortae, joining to form the descending aorta.

(Pictures have been slightly retouched to attenuate the grain of the $16 \mathrm{~mm}$ cine film frames.) 
A final diagnosis of isolated interruption of the aortic arch was made. An operation was contemplated in order to replace the absent arch, but the patient refused.

\section{Discussion}

The different locations of the interrupted aortic arch have provided an anatomical basis for the classification of this anomaly. To the first two types described by Abbott (1936), Celoria and Patton (I959) added a third. In type $A$, interruption of the arch occurs just distal to the left subclavian artery, and it behaves as an extreme coarctation of the aorta in its usual location. The embryological mechanism involved would be the abnormal involution of the dorsal aorta between the 4th and 6th arches, at about the 7 th week of embryonic development, i.e. after the normal progression of the left subclavian artery to its final position.

Type $B$ is characterized by the interruption of the arch between the left common carotid and the left subclavian arteries. In this case, involution would affect the 4th left aortic arch and the segment of dorsal aorta between the 4 th and 6th arches. This would happen well before the seventh week, so that the left subclavian artery could progress cranially only up to the level of the 6th arch.

In type $\mathrm{C}$ the arch is interrupted between both common carotid arteries; its embryogenesis is perhaps more complex: involution of the left side of the aortic sac would cause the absence of the $4^{\text {th }}$ arch and the ventral aspect of the 3 rd, while the dorsal aorta would persist between the 3 rd and 4 th arches.

All these types have been further subdivided, according to the dimensions of the persistent ductus, to the origin of the right subclavian artery, and even to the coexistence of an aortopulmonary window or a persistent truncus arteriosus ( $\mathrm{McNamara}$ and Rosenberg, I968; Moller and Edwards, I965). Usually, a large variety of associated cardiac defects is reported; a ventricular septal defect is the most frequent, more than 80 per cent of the group (Lie, I967). Of course, all of them keep patent a ductus arteriosus which allows the blood flow to reach the descending aorta. Only three previous reports of interruption of the aortic arch without a ductus or any other cardiac abnormality have come to our attention (Pillsbury et al., I964; Zetterquist, I967; Morgan et al., I970). There is also a report (Evans, I933) of a necropsy finding of atresia of the aortic arch without a ductus or a ventricular septal defect. This case is a type A (Celoria and Patton, I959); those of Morgan and Zetterquist have the anatomy of type B; Pillsbury's patient is a type C.

The anatomy of the patient here reported corresponds to a type $B$, associated with an aberrant right subclavian artery (Fig. 2). The latter is due to the interruption of the 4th aortic arch and right dorsal aorta between the $4^{\text {th }}$ arch and the 7 th segmental artery before its normal cranial translation. The distal confluence of both dorsal aortae give origin to the descending aorta; we have already detailed the general embryogenesis of this type.

The haemodynamics of this anomaly can be deduced from the angiographic time course and anatomical details. The full left ventricular output is received by the common carotid arteries; a part of this flow reaches the basilar system through the internal carotid and provides retrograde filling to both vertebral arteries. A major part of the retrograde flow, however, is provided by a large collateral arterial system, probably through normal preexistent vessels belonging to the complex network of branches of the external carotid; the most likely common distal pathway appears to be the thyrocervical trunk, which normally arises from the subclavian artery; its flow, in this situation, would be reversed.

Little is known of the mechanisms responsible for the interruption of the arch. It is a widespread belief that the pattern of flow through the arch in the embryonic stage can influence the type of the final abnormality. Congdon (1922) suggested that atresia of the arch would result if the separation of flow to head and body were performed through the 3 rd and 6th arches, instead of the normal pathway through the 3rd and 4th. Kleinerman et al. (1958) introduce the possibility of an abnormal septation of the truncus as a cause of aortic hypoplasia, leading eventually to atresia of the fourth arch. In those cases associated with a ventricular septal defect Van Praagh et al. (I97I) believe that the abnormal leftward displacement of the crista supraventricularis would induce, in succession, a subpulmonary septal defect, obstruction of the left ventricular outflow, and secondary atresia of the aortic arch. None of these mechanisms seems to be acceptable in the type reported here, as no other cardiac malformations are present. Neither were we able to show any disturbance in the metabolism of calcium.

An interesting question concerns the genesis of the collateral cervical vessels. There can be little doubt that they were developing well before the closure of the ductus; and that they were already functioning before birth. We believe them to be true collateral vessels developed from branches of the external carotid, joining with branches of the thyrocervical trunk, but the doubt arises whether these vessels might represent a persistence of the more cranial segments of the dorsal aorta.

The diagnosis of this condition should be suspected whenever the carotid pulses are well felt, but 

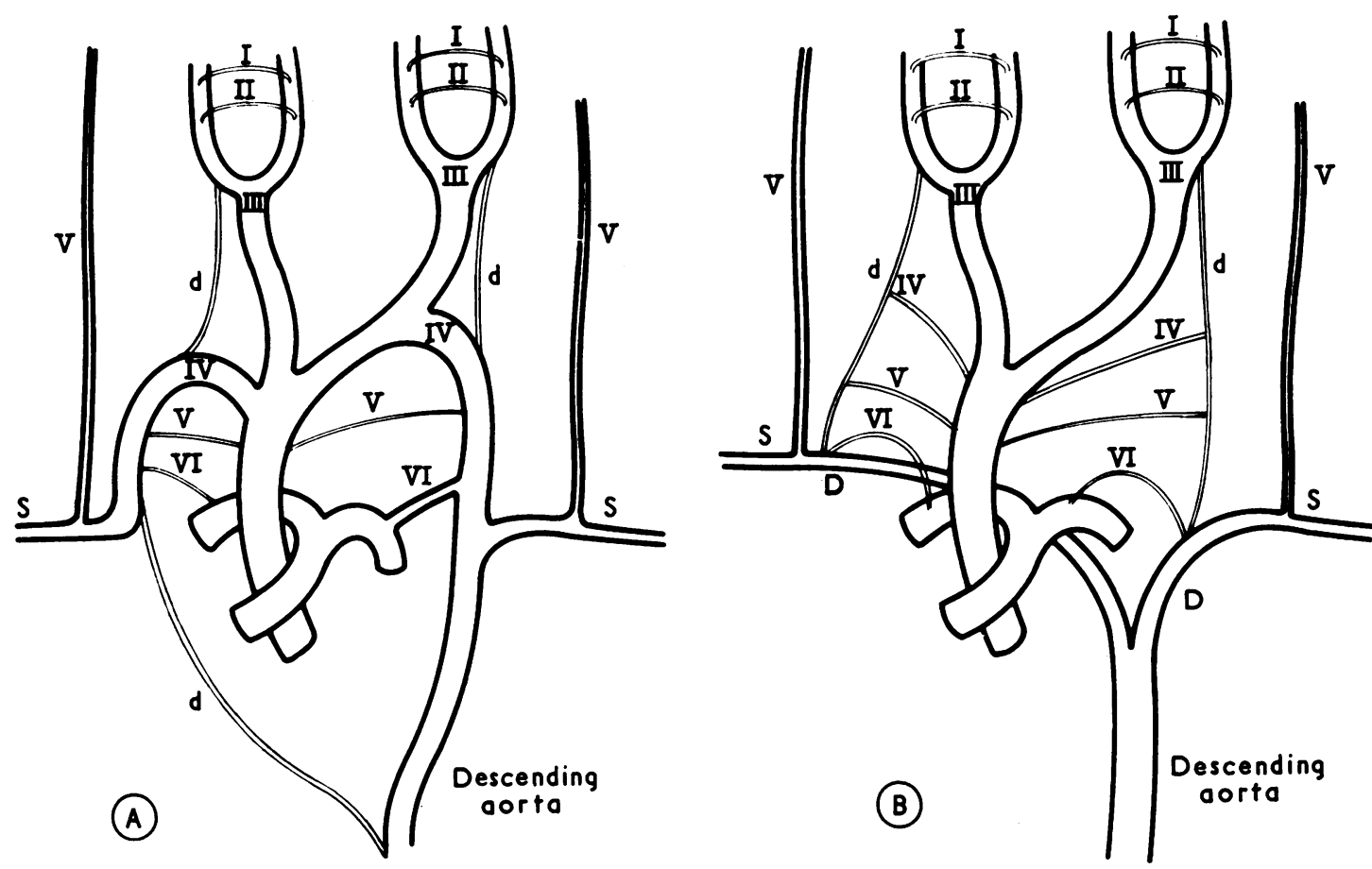

FIG. 2 (A) Schematic diagram of the normal aortic arch development. The left subclavian artery is illustrated before its cranial displacement to its final position proximal to the isthmus. $(B)$ Suggested embryogenesis of the reported case. Disappearing structures are drawn in light trace. I, II, III, IV, V, VI: aortic arches; V: vertebral artery; S: subclavian artery; $D$ : dorsal aortae, persistent in the present case; $d$ : involuted dorsal aortae.

no other arteries are detectable at the periphery. This must be differentiated from coarctation of the aorta, with an anomaly of origin, course, or ostial size of both subclavian arteries (Nathoo, 1965). The angiographic appearance should be diagnostic. The precise anatomical distinction between interruption and atresia of the arch, however, needs direct observation; the presence of a fibrous tract joining the blind ends of the ascending and descending aorta typifies atresia of the arch (Tawes et al., 1969; Moller and Edwards, 1965). Morgan et al. (1970) suggest that a definite posterior incurvation of the ascending aorta would indicate the presence of this fibrous tract, pulling it dorsally. In our case, the aortic bending was not conclusive and the refusal of the patient to have surgical treatment prevented us from establishing this point.

We accept, then, that the present case is the fourth reported instance of interrupted aortic arch without associated cardiac abnormalities, and consider that its clinical and anatomical data correspond closely to those of the patient reported by Morgan et al. (1970). It is of interest that the four reported cases have reached adult life without apparent haemodynamic embarrassment, in complete contrast to the poor outlook of most cases in which there was a persistent ductus or other congenital defect; that implies that the life expectancy is determined by the associated anomalies, and that the interruption itself is not inconsistent with a fair prognosis.

\section{References}

Abbott, M. E. (1936). Atlas of Congenital Cardiac Disease, p. 50. American Heart Association, New York.

Celoria, G. C., and Patton, R. B. (1959). Congenital absence of the aortic arch. American Heart fournal, 58, 407.

Congdon, E. D. (I922). Transformation of the aortic-arch system during the development of the human embryo. Contributions to Embryology, Carnegie Institution, 14, 47.

Evans, W. (1933). Congenital stenosis (coarctation), atresia, and interruption of the aortic arch. Study of 28 cases. Quarterly fournal of Medicine, 26, $\mathrm{x}$.

Kleinerman, J., Yang, W. M., Hackel, D. B., and Kaufman, N. (1958). Absence of the transverse aortic arch. Archives of Pathology, 65, 490. 
Lie, J. T. (1967). The malformation complex of the absence of the arch of the aorta (Steidele's complex). American Heart fournal, 73, 615.

McNamara, D. G., and Rosenberg, H. S. (1968). Interruption of the aortic arch. In Paediatric Cardiology, p. 224. Ed. by Hamish Watson. Lloyd-Luke, London.

Moller, J. H., and Edwards, J. E. (I965). Interruption of the aortic arch. Anatomic patterns and associated cardiac malformations. American fournal of Roentgenology, 95, 557.

Morgan, J. R., Forker, A. D., Fosburg, R. G., Neugebauer, M. K., Rogers, A. K., and Bemiller, C. R. (1970). Interruption of the aortic arch without a patent ductus arteriosus. Circulation, 42, 96r.

Nathoo, A. R. (1965). Two unusual cases of coarctation of the aorta. Guy's Hospital Report, 114, 32.
Pillsbury, R. C., Lower, R. R., and Shumway, N. E. (1964). Atresia of the aortic arch. Circulation, 30, 749.

Tawes, R. L., Panagopoulos, P., Aberdeen, E., Waterston, D. J., and Bonham-Carter, R. E. (1969). Aortic arch atresia and interruption of the aortic arch: experience in II cases of operation. Fournal of Thoracic and Cardiovascular Surgery, 58, 492.

Van Praagh, R., Bernhard, W. F., Rosenthal, A., Parisi, L. F., and Fyler, D. C. (197I). Interrupted aortic arch. Surgical treatment. American fournal of Cardiology, 27, 200.

Zetterquist, P. (1967). Atypical coarctation of the aorta with bilateral vertebral-subclavian pathway. Scandinavian Fournal of Thoracic and Cardiovascular Surgery, 1, 68.

Requests for reprints to V. M. Judez, Clinica Puerta de Hierro, S. Martin de Porres 4, Madrid 35, Spain. 